Check for updates

Cite this: RSC Adv., 2018, 8, 32522

Received 11th August 2018

Accepted 13th September 2018

DOI: $10.1039 / \mathrm{c} 8 \mathrm{ra06741d}$

rsc.li/rsc-advances

\section{A simple strategy for converting starch to novel compressible carbonaceous foam: mechanism, enlightenment and potential application $\dagger$}

\begin{abstract}
Hong Lei, (D)* Yao Wu, Sen Yang, Chunfang Fu and Jichuan Huo (DD *
Preparation of new materials from biomass is good for sustainable development. Carbon/carbonaceous foams (CFs), as important materials widely applied in both military and civil use, are usually made from nonrenewable materials at high temperature (usually over $1000{ }^{\circ} \mathrm{C}$ ) and high pressure (at $\mathrm{MPa}$ magnitude). Although some biomass raw materials have been used, there is still a fascinating issue waiting to be discussed. That is, what kind of biomass would be suitable for making CFs, or what the biggest challenge might be if these raw materials are used. Herein, a CF with advanced compressibility was made from starch with a simple and energy efficient strategy. The product can be made at much lower temperature (below $500{ }^{\circ} \mathrm{C}$ ) and pressure ( 190 Pa) than conventional strategies. The understanding of the conversion mechanism provides inspiration for the conversion of other biomass into value-added carbonaceous materials. Due to the presence of reactive groups on the surface of the as-prepared CF, the product can be further modified or used as a substrate to prepare composites for catalysis, phase change energy storage, sensors, water purification, and so on. In this work, its potential application in oil/water separation was demonstrated.
\end{abstract}

\section{Introduction}

Under the pressure of shortages of non-renewable resources, preparation of various new materials from renewable biomass is a good choice for sustainable development. Carbon/ carbonaceous foams (CFs) are important materials in addition to other forms of carbon materials such as carbon nanotubes, grapheme and graphitized nanocarbons. ${ }^{1-4}$ With 3D sponge like structures, they have been widely applied for both military and civil use, such as electrode materials, adsorption, vibration damping and impact or sound absorption, catalyst carrier, heat exchanger, electromagnetic shielding, radar absorption, filtration, aerospace material, etc. ${ }^{5,6}$ They have drawn far-ranging attention from many research institutions including the US Air Force Research Laboratory (AFRL), Oakridge National Laboratory (ORNL), the US Navy and NASA. ${ }^{7}$ Many new

State Key Laboratory for Environment-friendly Energy Materials, School of Materials Science and Engineering, Southwest University of Science and Technology, Mianyang 621010, People's Republic of China.E-mail: honglei117@163.com; huojichuan@ swust.edu.cn

$\dagger$ Electronic supplementary information (ESI) available: Digital images of the samples during the transition from starch to carbonaceous foam. SEM image of native corn starch. The molecular weight distributions of the native corn starch and the dark brown glutinous intermediate formed during the preparation of carbonaceous foam. Digital images of the carbonaceous products prepared from the starch filter cakes that were hydrolytically etched by acid mixture. Supplementary videos showing the mechanic property of the material and oil/water separation. See DOI: 10.1039/c8ra06741d strategies have been developed to prepare CFs in recent years. However, the most used carbon sources are still some nonrenewable precursors like mesophase pitch, coal tar pitches or synthetic resin. ${ }^{\mathbf{8 - 1 0}}$ In some reports, sucrose and tannin have been adopted. ${ }^{11-17}$ In addition to these biomass materials, polysaccharides such as starch, cellulose and chitosan, are very attractive due to their huge reserves. As the largest group of polymers produced in the world, the annual production of polysaccharides is about $150000 \mathrm{M}$ tons. ${ }^{18}$ However, the reports on CFs directly prepared from polysaccharides are limited, ${ }^{19-22}$ not to mention from the other biomass materials also with huge reserves.

We can't help wondering why so few people use biomass to make CFs. The answer to this question may be varied. But it is certain that the understanding of the conversion mechanism from biomass to CFs is absolutely important before answering this question. Although the formation mechanism from mesophase pitch to CFs has been reported, ${ }^{23}$ it is not sure whether this mechanism is applicable to biomass or not. There have been some reports on biomass derived CFs. However, there is a fascinating issue still waiting to be discussed. The issue is what kind of biomass would be suitable for making CFs, or what the biggest challenge might be if these raw materials are used. Considering the huge reserves of biomass, it is meaningful to figure it out.

From the application point of view, when CFs are used as composite matrices, it is desirable that the CFs have many reactive groups on their surface in addition to good mechanical 
strength or toughness. Conventionally, most of the CFs are prepared at high temperatures (usually over $1000{ }^{\circ} \mathrm{C}$ ) and high pressures (at MPa magnitude).$^{24}$ Vitreous CFs and graphitic CFs may be obtained depending on the preparation conditions. They are usually brittle and have few reactive groups on their surface, which is detrimental to further modification and use in composite preparation. In this work, we prepared $\mathrm{CF}$ at relatively lower temperature (below $500{ }^{\circ} \mathrm{C}$ ) and much lower pressure ( 190 Pa). The as-prepared CF is compressible and has abundant reactive groups on its surface, which is good for further modification. Our latest research shows that this CF has comparable thermal conductivity to the conventional graphitic CFs obtained at $1000{ }^{\circ} \mathrm{C}^{.24}$ It has potential to be applied in catalysis, phase change energy storage, sensing, water purification and other fields. The related work will be published elsewhere.

The current goal of this work is mainly on the formation mechanism of the starch-derived CF. The research shows that the transition from starch to $\mathrm{CF}$ is much similar to the formation of polymer foam. Proper pressure, exhaust and heat transfer are the keys to making well-shaped product. The research is valuable not only for the use of starch, but also for converting other biomass into value-added carbonaceous materials. Finally, the starch derived CF was surperhydrophobically modified (water contact angle: $167^{\circ}$ ) and its potential application in oil/water separation was demonstrated.

\section{Materials and methods}

\section{Materials}

Cornstarch (amylose content, 25\%) was obtained from Xi'an Xiadian Corn Development Co., Ltd (Shanxi, China). Concentrated sulfuric acid (98\%), phosphoric acid (85\%), $n$-hexane (AR) were purchased from Chengdu KeLong Chemical Co., Ltd (Sichuan, China). Nickel foam (thickness $1.5 \mathrm{~mm}$, surface density $350 \pm 20 \mathrm{~g} \mathrm{~m}^{-2}$, PPI 110) was bought from Changsha Liyuan New Material Co., Ltd (Hunan, China). Hexamethyl disilylamine (HMDS) was supplied by Aladdin Industrial Corporation (Shanghai, China). In order to clearly demonstrate the oil/water separation, the distilled water used for oil/water separation was dyed by methyl orange (Chengdu KeLong Chemical Co., Ltd, Sichuan, China).

\section{Experimental procedure}

The transition mechanism from starch to $\mathrm{CF}$. In a typical preparation, $5 \mathrm{~g}$ of corn starch was dispersed in $50 \mathrm{~mL}$ of aqueous acid mixture, which was prepared by mixing $5 \mathrm{~mL}$ sulfuric acid $\left(1 \mathrm{~mol} \mathrm{~L}^{-1}\right), 25 \mathrm{~mL}$ of phosphoric acid $\left(1 \mathrm{~mol} \mathrm{~L}^{-1}\right)$ and $20 \mathrm{~mL}$ of distilled water. The dispersion was immediately filtered with vacuum filtration. A quartz tube was set on the Buchner funnel firstly before filtration. The starch dispersion was then poured into the quartz tube and filtered. The aim of this operation is to obtain a filter cake with the same diameter of the quartz tube. The quartz tube has inner diameter of $5 \mathrm{~cm}$ and height of $10 \mathrm{~cm}$. It was used as a mold for the subsequent preparation of CF. The filter cake together with the filter paper was placed on a ceramic plate after filtration. A piece of nickel foam with diameter of $5 \mathrm{~cm}$ was then place on the top of the filter cake, so as to evenly disperse the force loading on the starch cake and create passages for exhaust. The abovementioned quartz tube, a beaker and a weight of $100 \mathrm{~g}$ were assembled as Fig. 1a. The assembled apparatus was then placed in an electric blast oven (GZX-9140MBE, Shanghai Boxun Industry \& Commerce Co., Ltd). The outlet of the hot air is at the bottom of the oven and the temperature change is shown as the inset of Fig. 1a. The temperature at the assembled apparatus was calibrated by a K-type thermocouple. It took $1 \mathrm{~h}$ to reach $180{ }^{\circ} \mathrm{C}$ and then maintained at this temperature for another $1 \mathrm{~h}$. Finally, the CF was obtained by taking the apparatus out of the oven, cooling to ambient temperature and removing the carbonaceous product from the mold.

The transition from starch to $\mathrm{CF}$ was investigated by collecting samples at $45 \mathrm{~min}, 55 \mathrm{~min}, 1 \mathrm{~h}, 2 \mathrm{~h}$ and $3 \mathrm{~h}$ after the mold being set in the oven. Their appearance and micromorphology were recorded by digital camera and Scanning Electron Microscopy (SEM), respectively. The melting behavior of the acid mixture impregnated corn starch and native corn starch was studied by Thermogravimetric-Differential Scanning Calorimetry (TG-DSC). The changes in functional groups during the transition were determined by Fourier Transform Infrared Spectroscopy (FTIR).

The role of acids and moisture. In order to study the effect of acids on the preparation of $\mathrm{CF}$, two starch dispersions were respectively prepared: (i) starch dispersed in distilled water and (ii) starch dispersed in acid mixture. For the starch dispersed in distilled water, its filter cake was set in the mold directly and subjected to foaming and carbonization as the typical preparation procedure. For the starch dispersed in acid mixture. The dispersion was set in an oven at $50{ }^{\circ} \mathrm{C}$ for $2 \mathrm{~h}$, and then the filter cake was soaked and washed by distilled water repeatedly to remove the acids. Finally, the filter cake without acids was set in the mold and subjected to foaming and carbonization. To figure out the role of moisture, the filter cake with acids (prepared as that in the above section) was kept in an oven at $60{ }^{\circ} \mathrm{C}$ for $24 \mathrm{~h}$ to remove moisture before foaming and carbonization.

The effect of hydrolytic etching. It is known that starch can be hydrolyzed in acidic environment. Considering that this may affect the crystallinity of the starch and further affect the preparation of CFs, the starch dispersed in acid mixture was set in an oven at $50{ }^{\circ} \mathrm{C}$ for 1,2 and $3 \mathrm{~h}$, respectively. Then the dispersion was filtered without washing by distilled water. Finally, the pre-hydrolyzed starch cakes were used to prepare CFs.

The effect of pressure, exhaust and heat transfer. We noticed that the pressure over the starch cake, the way of exhaust and the direction of heat flow have great effect on the shape of the final carbonaceous product. In order to obtain well-shaped CF, following four experiments were conducted. The starch cakes used in these experiments were all prepared by dispersing starch in acid mixture and keeping the dispersion in an oven at $50{ }^{\circ} \mathrm{C}$ for $1 \mathrm{~h}$ before filtration. 
(a)

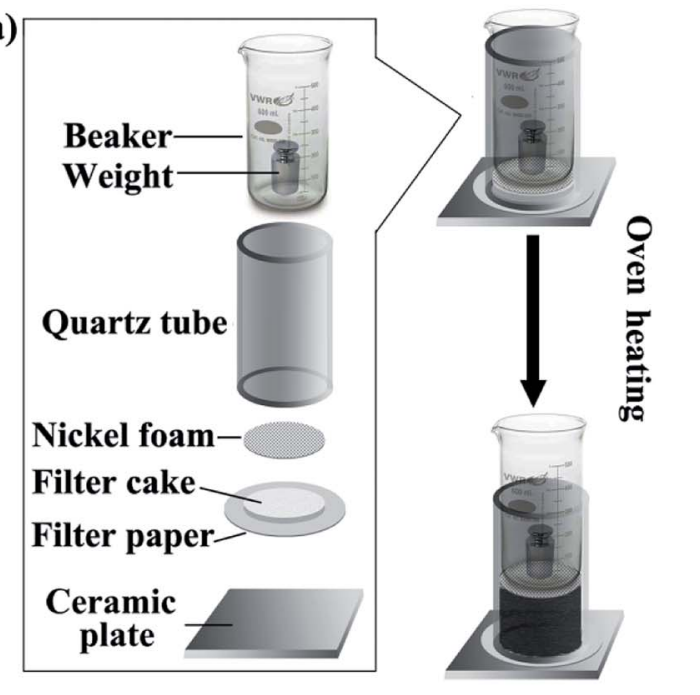

(b)

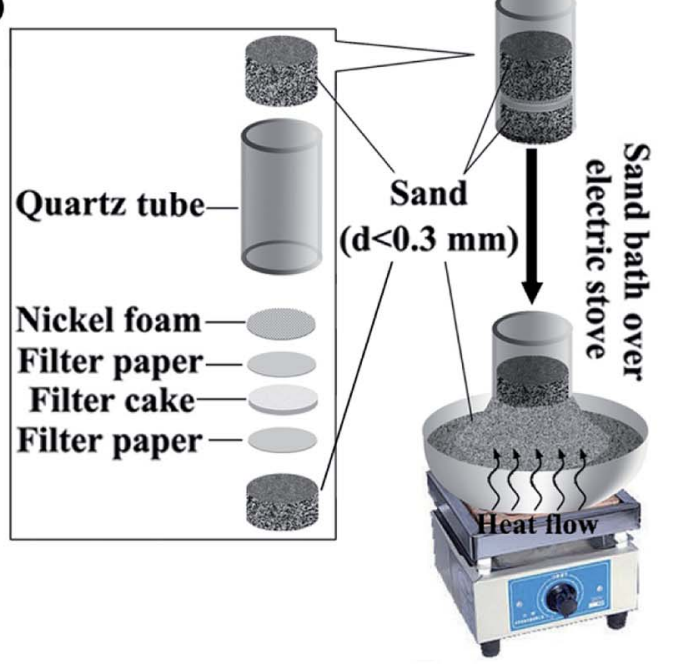

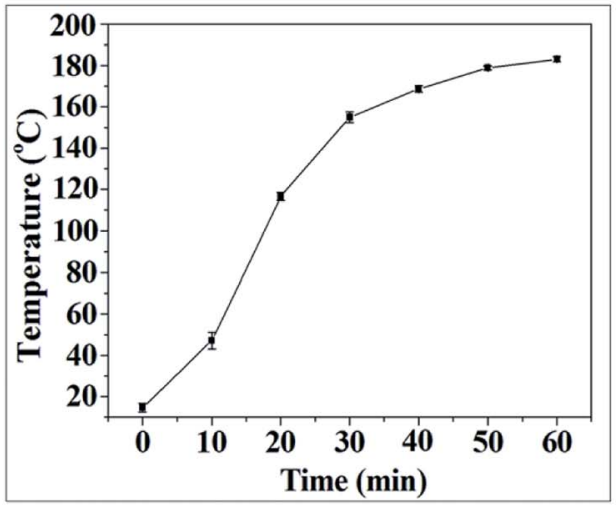

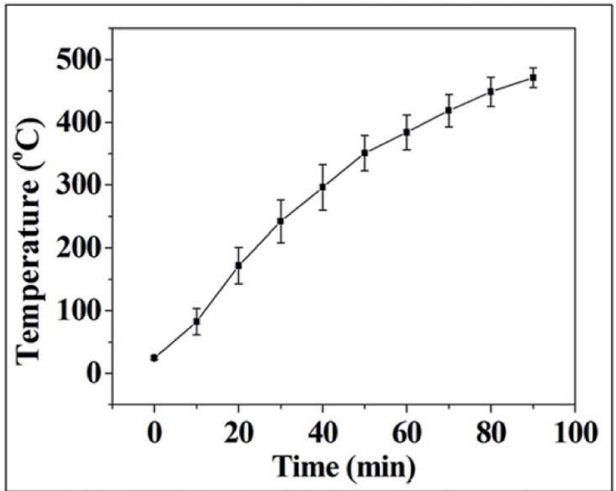

Fig. 1 Schematic diagram of preparing CF from starch: (a) prepared with electric blast oven, (b) prepared with a sand bath heated by electric stove.

(i) The starch cake was placed on a ceramic plate and heated in the electric blast oven (GZX-9140MBE) whose outlet of hot air is at the bottom.

(ii) CF was prepared by using the apparatus in Fig. 1a with electric blast oven GZX-9140MBE.

(iii) CF was prepared by using the apparatus in Fig. 1a, but with another electric blast oven whose outlet of hot air is located on the back of the oven (DHG-9070A, Shanghai Keelrein Scientific Instrument Co., Ltd).

(iv) CF was prepared using a sand bath heated by electric stove (Fig. 1b). In short, part of the quartz tube was vertically inserted in the sand bath firstly. Then, the starch cake sandwiched between two filter papers was place in the tube. A piece of nickel foam was subsequently placed on the top and $150 \mathrm{~g}$ of sand was poured over the nickel foam. Finally, the sand bath was heated by the electric stove. After $90 \mathrm{~min}$ of heat treatment, the CF was obtained by removing it from the mold. It is note that $150 \mathrm{~g}$ of sand was applied here because we considered the weight of the beaker in (ii) and (iii), which corresponds to a pressure of about $190 \mathrm{~Pa}$ on the starch cake. This pressure is much lower than that required in conventional strategies (usually at the magnitude of MPa).

Modification and oil/water separation. The surperhydrophobic CF was prepared by simply immersing the obtained $\mathrm{CF}$ in HMDS for $12 \mathrm{~h}$ and then oven-dried at $60^{\circ} \mathrm{C}$. For the sample used for oil/water separation, the obtained CF was cut into the desired shape before immersion in HMDS.

\section{Characterization}

SEM (TM-1000, HITACHI, Japan; Ultra 55, Carl Zeiss SMT Pte Ltd, Germany) was used to observe the morphology of the product. TG-DSC measurement was conducted on a Q200 (TA instruments, US) in nitrogen flow (flow rate: $50 \mathrm{~cm}^{3} \mathrm{~min}^{-1}$, heating ramp: $5{ }^{\circ} \mathrm{C} \mathrm{min}^{-1}$ ). FTIR spectra were measured on a Nicolet 380 (Thermo Fisher Scientific) spectrometer using $\mathrm{KBr}$ pellets. The molecular weight distributions of the raw 

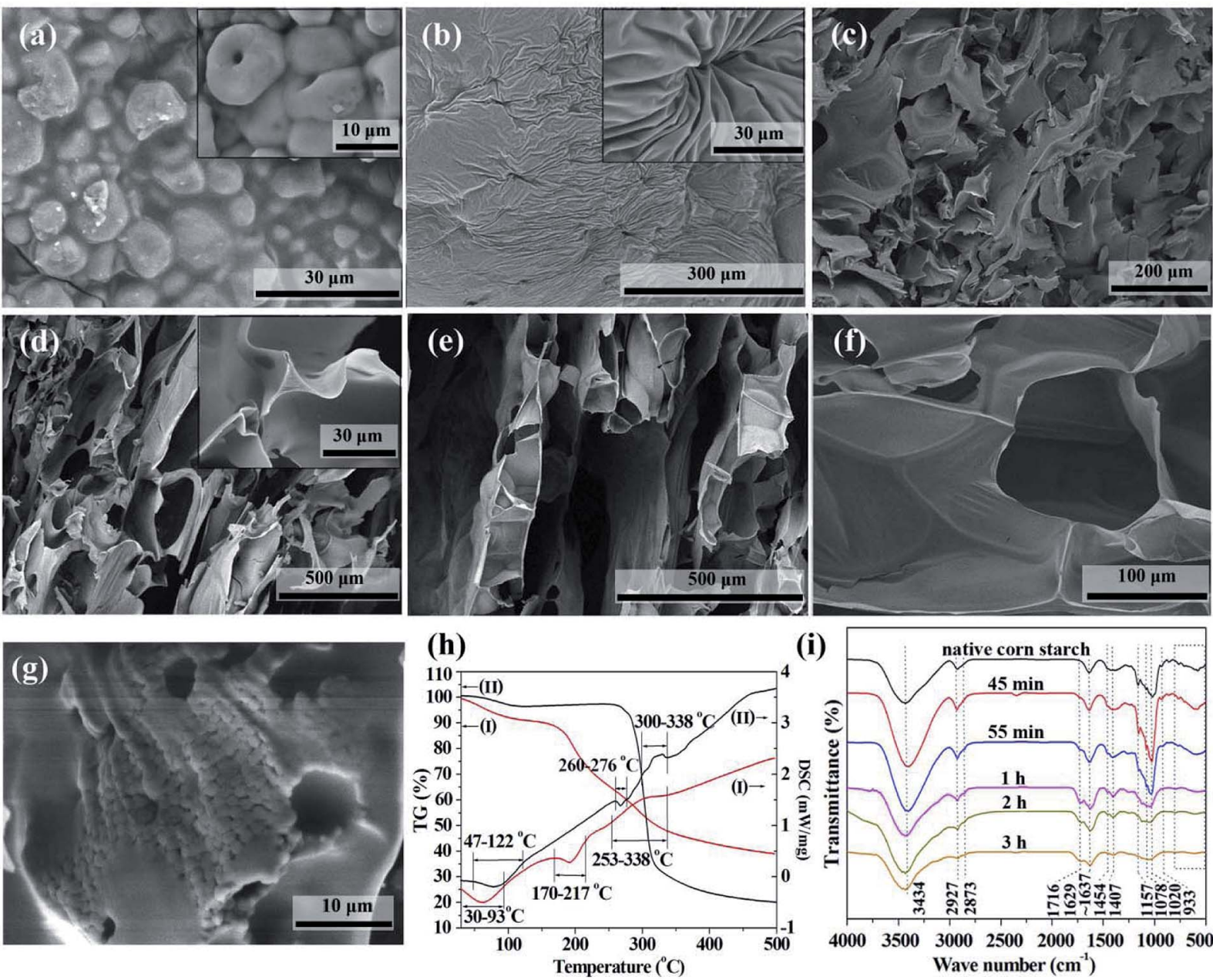

(i)

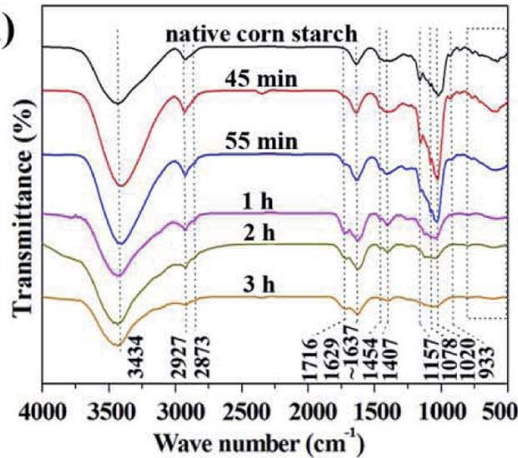

Fig. 2 SEM images of the samples during the transition from starch to CF: (a) 45 min, (b) and (c) 55 min, (d) $1 \mathrm{~h}$, (e) $2 \mathrm{~h}$ and (f) 3 h; (g) carbonized starch grains before completely hydrolysis; (h) TG and DSC curves of (I) the acid mixture impregnated corn starch and (II) the native corn starch; (i) FTIR spectra of native corn starch and the intermediate during the conversion of starch to CF.

material (native corn starch) and the intermediate during the formation of CF were analyzed by a multi-angle laser light scattering gel permeation chromatography (MALS/GPC (SEC), Agilent 1200 and Wyatt DAWN series) with aqueous mobile phase containing $0.1 \mathrm{~mol} \mathrm{~L}^{-1}$ of $\mathrm{NaNO}_{3}$ and $0.02 \mathrm{wt} \%$ of $\mathrm{NaN}_{3}$. The aqueous solutions containing native starch molecules $\left(5 \mathrm{mg} \mathrm{mL}{ }^{-1}\right)$ and intermediate material $\left(1 \mathrm{mg} \mathrm{mL}^{-1}\right)$ were filtered through a Nylon 66 syringe filter $(0.22 \mu \mathrm{m})$ respectively before injection. X-ray diffraction (XRD) patterns were recorded on an X'Pert PRO (PANalytical B.V.) equipped with $\mathrm{Cu} \mathrm{K} \alpha$ $\mathrm{X}$-ray source $(\lambda=0.154 \mathrm{~nm})$ operated at $40 \mathrm{kV}$ and $40 \mathrm{~mA}$. Elemental analysis was conducted a Vario EL CUBE CHNS Analyzer (ELEMENTAR, Germany). The oxygen content is calculated using a depletion method after $\mathrm{C}, \mathrm{H}, \mathrm{N}$ and $\mathrm{S}$ values. The compression property of the $\mathrm{CF}$ was investigated by a C45.504 universal testing machine (MTS Industrial Systems Co., Ltd China). Static water contact angles were measured using a drop shape analyzer (DSA30, Krüss, Germany) with droplet volume of $2.5 \mu \mathrm{L}$.

\section{Results and discussion}

\section{The transition mechanism from starch to $\mathrm{CF}$}

After the sample was placed in the oven with elevating temperature, its color changed from the white of starch to the light yellow and then dark brown of some glutinous material and finally to the black of carbonaceous product (Fig. S1 $\dagger$ ). The transition from starch to carbonaceous product took place at about $55 \mathrm{~min}$ in our case, at which time the temperature had just risen to about $180^{\circ} \mathrm{C}$. After that, the sample was gradually foamed, showing a higher sample height.

The SEM images of the native corn starch and samples during the transition from starch to CF are shown in Fig. S2 $\uparrow$ and 2. As shown in Fig. 2a, starch granules can still be observed at $45 \mathrm{~min}$, but their edges are fused together. The inset of Fig. $2 \mathrm{a}$ illustrates some granules with pores in them. This is the result of acid catalyzed hydrolysis of starch. When the starch granules were almost melted at $55 \mathrm{~min}$, these pores evolved further into the navels shown in Fig. 2b. In addition to this molten state of 
(a)

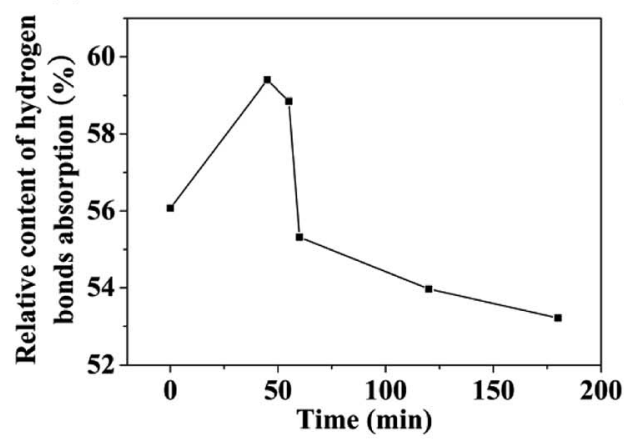

(c)

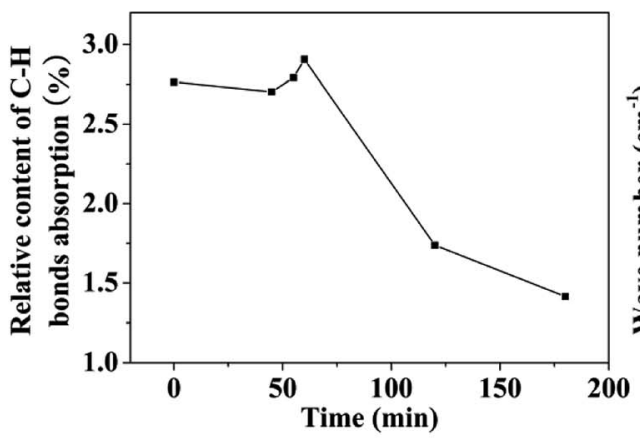

(b)

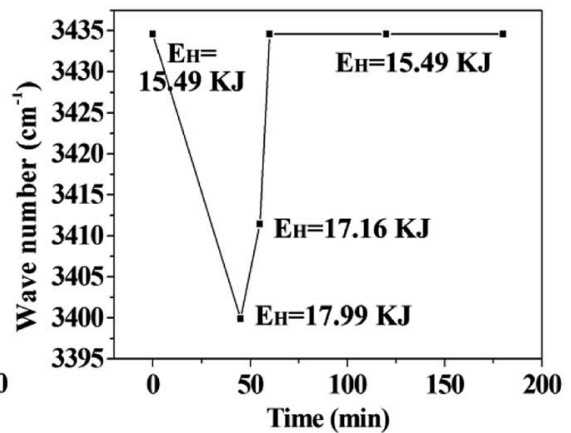

(d)

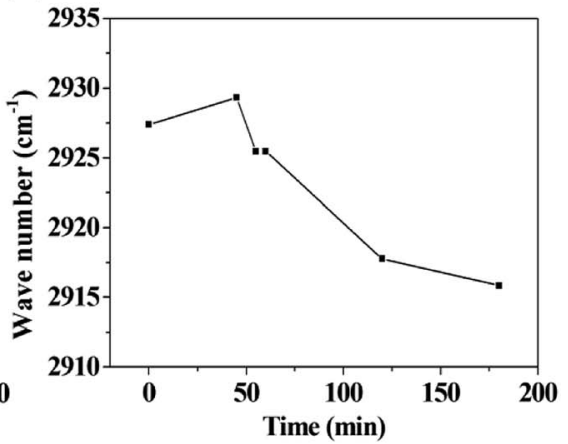

Fig. 3 FTIR peak change during the transition from starch to CF: (a) relative content of hydrogen bonds absorption (integrated from 3006 to $3737 \mathrm{~cm}^{-1}$ ); (b) frequency change of hydrogen bonds absorption and energy of hydrogen bonds; (c) relative content of $\mathrm{C}-\mathrm{H}$ bonds absorption (integrated from 2806 to $3005 \mathrm{~cm}^{-1}$ ); (d) frequency change of $\mathrm{C}-\mathrm{H}$ bonds absorption.

starch, some part of the sample locating around the edge of the cake transformed to carbonaceous sheets at 55 min (Fig. 2c). Because the heat transfers from the outside to the center of the cake, the edge of the cake evolved faster than the center. These sheets were further foamed and carbonized and finally became the carbonaceous product with ultrathin cell walls (Fig. 2d-f). According the height of the carbonaceous sample and the formation of triangle strut shown in the inset of Fig. $2 \mathrm{~d}$, the foaming process started at about $1 \mathrm{~h}$ after the sample was placed in the oven.

It is noteworthy that the molten state is a turning point in the conversion of starch to CF. Therefore, TG-DSC measurements were conducted to investigate the melting behavior of the acid mixture impregnated corn starch and native corn starch (Fig. 2h). The acid mixture impregnated corn starch used for TG-DSC measurement was prepared by drying the acidic solution impregnated starch cake in an oven at $60{ }^{\circ} \mathrm{C}$ for $24 \mathrm{~h}$. The DSC curves in Fig. $2 \mathrm{~h}$ show that both samples have two endothermic peaks and one exothermic peak. The endothermic peaks at the lower temperature region are due to the evaporation of adsorbed moisture. The TG curves corresponding to the endothermic peaks at the higher temperature region show that the samples have only a small amount of weight loss, indicating that the endothermic peaks are due to the melting of the samples. In contrast, the exothermic peaks of both samples correspond to a large proportion of weight loss, indicating that they are caused by the carbonization of the samples. In addition, the residual of acid mixture impregnated corn starch was significantly more than that of the native starch. This is a wellknown phenomenon that the addition of acids allows increasing the carbon yield of biomass. The result in Fig. $2 \mathrm{~h}$ also shows that the melting and carbonization of the samples are two separate processes. Foaming is most likely to happen between these two processes, where the TG curves are steep and decomposition gases are produced. The addition of acids reduced the melting temperature of starch and broadened the carbonization temperature interval. The broadened carbonization temperature interval allows the foamed structure of the intermediate to be carbonized and fixed in time. It also allows the $\mathrm{CF}$ to be prepared at much lower temperature than conventional methods, which typically operate at temperatures above $1000{ }^{\circ} \mathrm{C}$ in inert atmosphere.

The importance of the molten state for the preparation of foamed product can be further confirmed by the SEM image of the CF prepared from the moisture-removed starch cake. Fig. $2 \mathrm{~g}$ shows an SEM image of this carbonaceous product. The majority of this sample has been foamed. But some characteristic regions containing small starch grains are still observed. These grains $(c a .1 \mu \mathrm{m})$ are much smaller than the size of native starch granules $(c a .10-20 \mu \mathrm{m})$. According to the structure of starch, the acids preferably hydrolyze the amorphous regions of the starch granules firstly, where the structure is relatively looser. ${ }^{25}$ Then the more compact crystalline parts are also hydrolyzed and the starch cake is finally converted to molten state. The transition from grains to molten starch can be seen from the fused edges of these grains (Fig. $2 \mathrm{~g}$ ). In the case that 
the starch filter cake contains insufficient moisture, the starch is less hydrolyzed before being carbonized and the carbonization effect of the acids is relatively enhanced. Therefore, some unhydrolyzed starch grains were carbonized before converting into molten state and retained in the final carbonaceous product.

The changes in functional groups during the transition from starch to CF are reflected from the FTIR spectra in Fig. 2i. The association of hydrogen bonds is responded by the absorption at $\sim 3434 \mathrm{~cm}^{-1}$. The absorptions at $\sim 2927$ and $\sim 1454 \mathrm{~cm}^{-1}$ are caused by the $\mathrm{C}-\mathrm{H}$ stretch in methylene and the absorptions at $\sim 2873$ and $\sim 1407 \mathrm{~cm}^{-1}$ are the reflection of methyl. Area normalization method was used to calculate the relative content of hydrogen bonds absorption (3006-3737 $\mathrm{cm}^{-1}$ ) and $\mathrm{C}-\mathrm{H}$ bonds absorption (2806-3005 $\left.\mathrm{cm}^{-1}\right)$. The results as well as the change of peak positions are plotted in Fig. 3. We also calculated the energy of hydrogen bonds $\left(E_{\mathrm{H}}\right)$ according to the frequency of the bonded $\mathrm{OH}$ groups. The calculation follows the following formula, ${ }^{26}$ and the results are noted in Fig. $3 \mathrm{~b}$.

$$
E_{\mathrm{H}}=\frac{1}{k}\left(\frac{\nu_{0}-\nu}{\nu_{0}}\right)
$$

where $\nu_{0}$ is the standard frequency corresponding to free $\mathrm{OH}$ groups $\left(3650 \mathrm{~cm}^{-1}\right), \nu$ is the frequency of the bonded $\mathrm{OH}$ groups, and $k$ is a constant $\left(1 / k=2.625 \times 10^{2} \mathrm{~kJ}\right)$. These results indirectly reveal some details of the conversion from starch to CF.

During the conversion process, the hydrogen bonds are first strengthened, and then weakened, and finally remains unchanged. In conjunction with the observation of conversion process in Fig. 2 and the molecular weight test of the intermediate product in the next section, we consider that the energy enhancement of the hydrogen bonds is probably related to two factors. The one is acid-catalyzed hydrolysis leading to the destruction of starch molecular chains. The second is the insertion and binding of polar small molecules such as water and inorganic acids with the starch molecules. The shortened starch molecular chains are more susceptible to twisting during the melting and foaming stages, which in turn makes the starch hydroxyl groups closer and more favorable for dehydration and carbonization. We believe that the twisting of the molecular chains plays an important role in the structural reconstruction of the precursor and the morphologies of the final carbonaceous product. Our previous study on the preparation of carbonaceous products from new gelatinized starch and retrograded starch showed that the pre-organized structure of the precursors has effect on the microstructure of the carbonaceous product. ${ }^{27}$ Therefore, we predict that perhaps we can adjust the structure of the precursor by adjusting the hydrogen bonds interaction, and finally adjust the microstructure of the carbonaceous product. But this remains to be verified by more experimental results. It should be noted that although the energy of hydrogen bonding is reduced to the same level as the raw material after CF formation, this reduction is due to dehydration and carbonization. The environment of its hydrogen bonding should be different from the raw material.
With the progress of foaming and carbonization, the absorption of carbonyl at $1716 \mathrm{~cm}^{-1}$ appeared, while the absorption at $1637 \mathrm{~cm}^{-1}$ (scissoring of two $\mathrm{O}-\mathrm{H}$ bonds of absorbed water molecules) shifted to $1629 \mathrm{~cm}^{-1}$ (stretching vibration of $\mathrm{C}=\mathrm{C}$ ). It is notable that the characteristic absorptions of starch (C-O-C at 1157, 1078 and $1020 \mathrm{~cm}^{-1}$; skeletal mode vibration of $\alpha-1,4$-glycosidic linkage at $933 \mathrm{~cm}^{-1}$ ) disappeared with the progress of carbonization and foaming.

\section{The role of acids and moisture}

Generally, the intermolecular bonds of starch molecules will be broken down in the presence of water and heat. This process is known as gelatinization of starch, resulting in disorganized starch molecules. However, this effect of water was not obviously observed in the filter cake made by dispersing starch in distilled water. As shown in Fig. 4a and c, no gelatinization of starch happened and the starch granules are still monodisperse. This may be due to the fact that the limited content of water in the filter cake was mainly evaporated with the elevating temperature. Fig. 4b and d illustrates that neither foaming nor carbonization would be observed if we wash the acids out even though the starch was pretreated with acid mixture. Again, no gelatinization of starch was observed. But it is different from the filter cake made by dispersing starch in pure distilled water
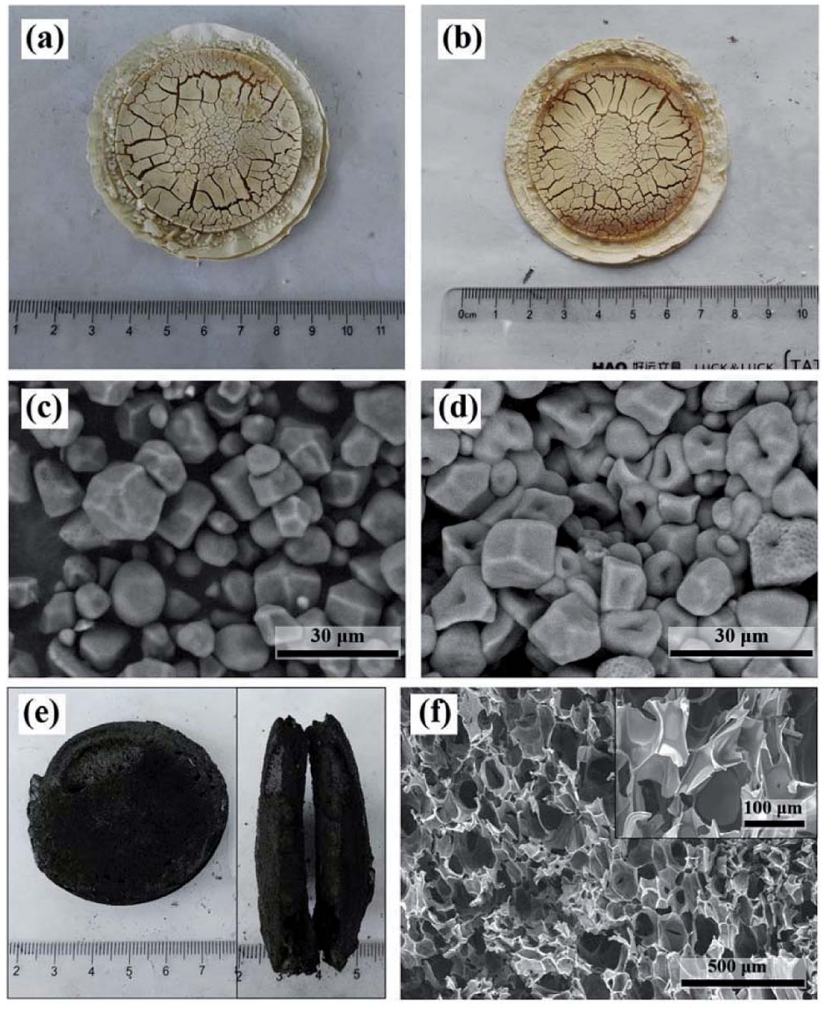

Fig. 4 (a) and (b) Digital images of unfoamed filter cakes made from starch dispersed in (a) distilled water and (b) acid mixture but washed with distilled water before foaming and carbonization; (c) and (d) SEM images of (a) and (b), respectively; (e) digital and (f) SEM images of the CF prepared from moisture-removed starch cake. 
(Fig. 4c), many pores caused by acid catalyzed hydrolysis were found in the acid-pretreated starch granules (Fig. 4d).

The result also indicates that the acid mixture played an important role in the formation of molten state at lower temperature for the acid-treated starch and further in the formation of CF. We believe that the formation of molten state at lower temperature is related to the continuous acid catalyzed hydrolysis of starch as well as the resulted disordered small molecules. To prove this, we measured the molecular weights of the native corn starch and the dark brown glutinous intermediate formed during the preparation of CF (Fig. S3 $\dagger$ ). The result indicates that the $M_{\mathrm{w}}$ of the native corn starch is $5.524 \times 10^{5} \mathrm{~g}$ $\mathrm{mol}^{-1}$ (mass fraction: $100 \%$ ), while the $M_{\mathrm{w}}$ of the dark brown glutinous intermediate is indeed much smaller $\left(9.244 \times 10^{3} \mathrm{~g}\right.$ $\mathrm{mol}^{-1}$ with mass fraction of $97.6 \%$ and $5.886 \times 10^{5} \mathrm{~g} \mathrm{~mol}^{-1}$ with mass fraction of $2.4 \%$ ). If the smaller molecules produced by hydrolysis are washed off with water, the fusion of starch granules will not be observed. That's exactly what we have seen in Fig. 4 d. The acid catalyzed hydrolysis can disrupt the crystal structure of starch. The hydrolyzed molecules accumulate around the starch granules and form disordered molten state to fuse the granules together. As the hydrolysis proceeds, all the starch granules eventually fuse and transform into a disordered molten state. This molten state is favorable for the even distribution of acids among the starch molecules, and thus is favorable to obtain a well foamed carbonaceous product.

Although the moisture does not contribute to the gelatinization of starch, it still plays an important role in the preparation of CF. As we have seen in Fig. $2 \mathrm{~g}$, the carbonization effect of the acids will be relatively enhanced if the starch filter cake contains insufficient moisture. As a result, a small amount of unhydrolyzed starch grains will be carbonized before converting into molten state and retained in the final carbonaceous product. Besides, compared with the CF prepared from starch filter cake containing moisture, the digital and SEM images in Fig. 4e and $\mathrm{f}$ show that the carbonaceous product from starch filter cake without moisture is more compact and the size of the cells is smaller (ca. $100 \mu \mathrm{m})$. Whereas the cell size of the former can reach to $300-700 \mu \mathrm{m}$. This also suggests that the carbonization effect of the acids was enhanced. The result shows a possibility of tailoring the foaming height and cell size by adjusting the moisture content in the filter cake.

In addition to the results in the above section, the study on the role of acids and moisture allows us to have a clearer understanding of the transition process from starch to CF than our previous study. ${ }^{28}$ This transition is much similar to the formation of polymer foam, which usually undergoes three stages: implementation of gas, expansion of gas, and stabilization of the polymer. ${ }^{29}$ The acids dispersion in the molten starch, decomposition gases release from the acids-starch reaction, and carbonization under the effect of acid dehydration are analogy to the above three stages, respectively. This suggests that we may be able to adjust the speed of foaming and carbonization by controlling the heating rate so as to produce $\mathrm{CF}$ with different porous structure. The above results also give us a profound revelation that if other renewable resources (such as cellulose, hemicellulose, lignin, and so on) can be made into molten state, then there is possibility to make them into CF, too. In fact, Wang et $a .^{30}$ has reported a method to prepare CF by submitting birch sawdust to liquefaction, resinification, foaming, carbonization, and activation steps, even though this study did not entirely use biomass feedstock as carbon source.

Interestingly, both starch and cellulose are composed of glucose units. The only difference in their chemical structure is that the glucose units in cellulose are linked by $\beta-1,4$ glycosidic bonds, while the glucose units in starch are linked by $\alpha$-1,4-glycosidic bonds and $\alpha$-1,6-glycosidic bonds. ${ }^{31} \mathrm{We}$ have tried to prepare CF from cellulose using the method in this work. However, the carbonized cellulose almost retained the morphological features of the raw material and was not foamed like starch. According to Turki et al. ${ }^{26}$ the energy of hydrogen bonds in cellulose is much higher than that in starch. Due to the strong hydrogen bonding in cellulose, polar small molecules such as water and inorganic acids are difficult to insert into its crystalline regions or to effectively catalyze the hydrolysis in a short period of time. Therefore, its molecular chains are unable to freely twist even when the dehydration and carbonization happens. This is why we usually do not see a melting endotherm in the DSC curve of cellulose. Although there is no foamed carbonaceous product obtained from cellulose, it again demonstrates that hydrogen bonding affects the reconstitution of the pre-organized structure of the precursors and ultimately affects the microstructure of the carbonaceous product.

Based on the above discussion and some reports on the preparation of CFs, the following methods may be selected to prepare CFs according to the characteristics of different biomass raw materials. When someone plans to prepare CFs from biomass feedstocks, having the feedstocks meet one of these characteristics may be the most challenging and critical step.

(i) For thermoplastic raw materials, CFs can be prepared by first foaming and then carbonizing. The foaming and carbonization may be carried out in a stepwise (just as the conventional strategy did, which using mesophase pitch as raw material) or synergistic manner (just as what we did in this work).

(ii) For raw materials composed of small molecule monomers, template method is applicable. Firstly, the monomers need to be deposited or polymerized in the gap of the template. Then, CFs can be obtained by removing the template and carbonizing the foam-like polymer or carbonizing the composite firstly and then removing the template.

(iii) For thermosetting raw materials, they need to have the foam-like structural features, or they can be converted to small molecules. For those with foam-like structural features, they can be directly carbonized to prepare CFs. ${ }^{32}$ If the raw material can be converted into small molecules, then the route (i) or (ii) may be effective depending on the small molecules are meltable or not.

(iv) For those raw materials already being carbonized, a postassembly method may be used to obtain CFs. A typical case of this method is the preparation of a graphene aerogel by freezedrying method. ${ }^{33}$ 


\section{The effect of hydrolytic etching}

Acidic hydrolysis usually creates pores in the starch granules and may cause changes in the crystallinity of the granules. This may affect the distribution of acids in the starch granules and further affect the formation of the molten state. Therefore, the effect of hydrolytic etching on the preparation of CF was investigated in this section. Different starch filter cakes that were hydrolytically etched by acid mixture for different times were used to prepare CFs. Shown in Fig. 5a-c are the densities, heights and yields of the obtained CFs. The yields here are defined as the mass percentage of the CFs to starch. It should be noted that some large cavities usually formed on the top of the products prepared with the electric blast oven GZX-9140MBE. The heights were measured after excision of the large cavities.

The results show that the starch can be foamed whether or not it is pre-hydrolyzed by acid or not, although the heights of the carbonaceous products are different. This is mainly because the heating process of the oven provides time for the hydrolysis and melting of the starch. According to Fig. 5b and c, the CF made from starch pre-hydrolyzed for $1 \mathrm{~h}$ has the highest height and yield. In contrast to the starch without pre-hydrolysis, more acids can be introduced into the partially hydrolyzed starch granules due to the formation of pores. Thus, the prehydrolyzed starch granules are more susceptible to further hydrolysis and melting. This results in a more homogeneous dispersion of acids in molten state and ultimately a better foamed product. However, prolonged pre-hydrolysis will reduce the yields. This is mainly because the hydrolyzed portion of the starch is dissolved in the liquid phase and then filtered into the filtrate, resulting in a reduction in the carbon source used to prepare CF. The densities in Fig. 5a have not changed much when the hydrolysis exceeds $1 \mathrm{~h}$. It suggests that the reduction in carbon source does not have much effect on the density of the carbonaceous product.

In order to understand the hydrolysis process of the starch in depth, the starch filter cakes pre-hydrolyzed for $0,1,2$ and $3 \mathrm{~h}$ were firstly washed with distilled water to neutral, then oven dried at $60^{\circ} \mathrm{C}$ and finally characterized by XRD. Fig. 5d shows a typical XRD pattern of the raw material (cornstarch). The peaks at $15,17,18$ and $23^{\circ}$ in $2 \theta$ are indicative of A-type crystallinity. The phase percentages of the starches were calculated using a modified Hermans method. ${ }^{34}$ Briefly, the crystalline, semi-crystalline and amorphous regions are divided on the XRD spectrum as Fig. 5d. The percentage of each phase is then
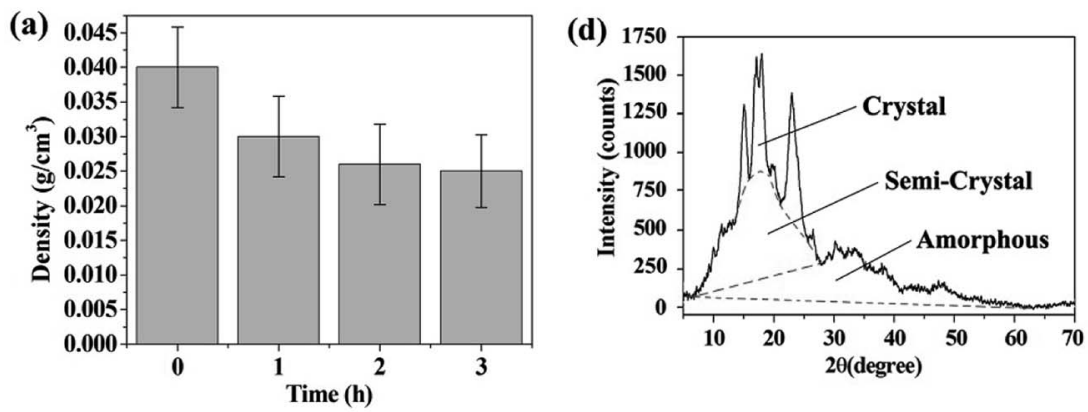

(b)

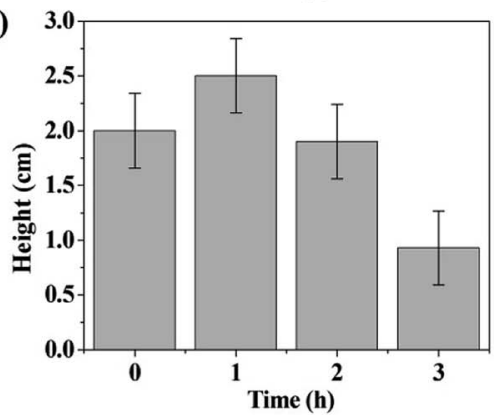

(e)

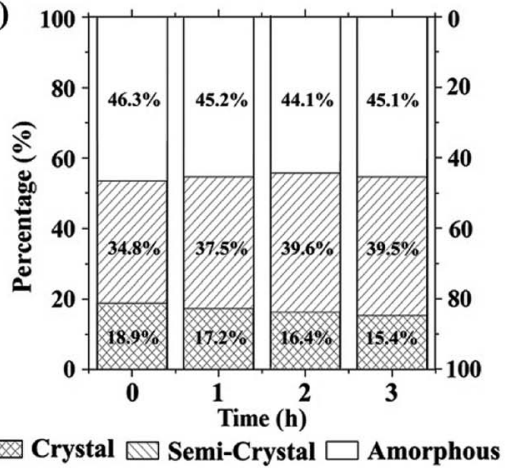

(c)

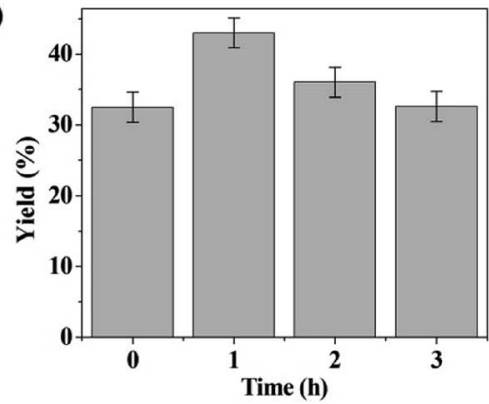

$\square$ Crystal $\square$ Semi-Crystal $\square$ Amorphous

Fig. 5 Effect of hydrolytic etching on the (a) density, (b) height and (c) yield of CF; (d) illustration of the phase percentage calculation in starch; (e) different phase percentages of the starches pre-hydrolyzed for 0, 1, 2 and $3 \mathrm{~h}$. 
obtained by dividing the corresponding area of the phase by the total area of the crystalline, semi-crystalline and amorphous regions. The results are shown in Fig. 5e.

With the increase of hydrolysis time, the percentage of amorphous phase shows the trend of decreasing at the beginning and then increasing. The initial percentage reduction of the amorphous phase may be related to the dissolution of the hydrolyzed molecules into the filtrate. As the hydrolysis continues, more and more amorphous phase formed, so that even if some of the hydrolyzed molecules are filtered out, the percentage of the amorphous phase in the filter cake increases. In addition, Fig. 5e shows that the percentage of the crystalline phase decreases and the semi-crystalline phase increases with the proceeding of hydrolysis. This indicates that the crystalline phase is first converted into a semi-crystalline phase having a lower degree of order, and then the semi-crystalline phase is further hydrolyzed into an amorphous component. This also suggests that the retained particles found in Fig. $2 \mathrm{~g}$ are probably derived from the highly ordered portion of the starch granules. They were carbonized before being completely converted to an amorphous molten state.

\section{The effect of pressure, exhaust and heat transfer}

Fig. 6 shows that the pressure over the starch cake, the way of exhaust and the direction of heat transfer have significant effect on the shape of the carbonaceous product. In Fig. 6a, nothing was set on the starch cake and the gas could be freely discharged from all directions. In Fig. 6b, a beaker and a weight were placed on the starch cake. In Fig. 6d, the beaker and weight were replaced with sand, which makes the exhaust more uniform in the vertical direction relative to the device in Fig. 6b. The shape of the carbonaceous product obtained in Fig. 6a, $\mathrm{b}$ and $\mathrm{d}$ indicates that proper pressure and gas venting obstruction are necessary to create a good shaped product. In addition to the pressure and way of exhaust, the influence of heat transfer cannot be ignored, too. Comparing Fig. 6b with Fig. $6 \mathrm{c}$, it can be found that the shape of the product is significantly different even if the heating program was the same but only used electric blast ovens with different heat flow direction. In the case where the hot air was blown from the side of the sample, the shape of the product is poor (Fig. 6c). There are many large cavities in the body of the product. This phenomenon may be related to the heating sequence of different parts of the starch cake. The first heated part will reach the foaming temperature sooner. If different parts of the starch cake simultaneously reach the foaming temperature, some small bubbles will merge with each other to form large cavities. However, if different parts of the starch cake are heated in a gradient, the first foamed part will be carbonized to stabilize the cell structure when the adjacent part is foamed.

We also found that the product in Fig. 6b usually has a large cavity in its upper part despite its good shape in the lower part. In contrast, the shape of the product in Fig. $6 \mathrm{~d}$ is much better. Besides, the product in Fig. $6 \mathrm{~b}$ is softer than the product in Fig. 6d. Their SEM images in Fig. 6e and f clearly show that the

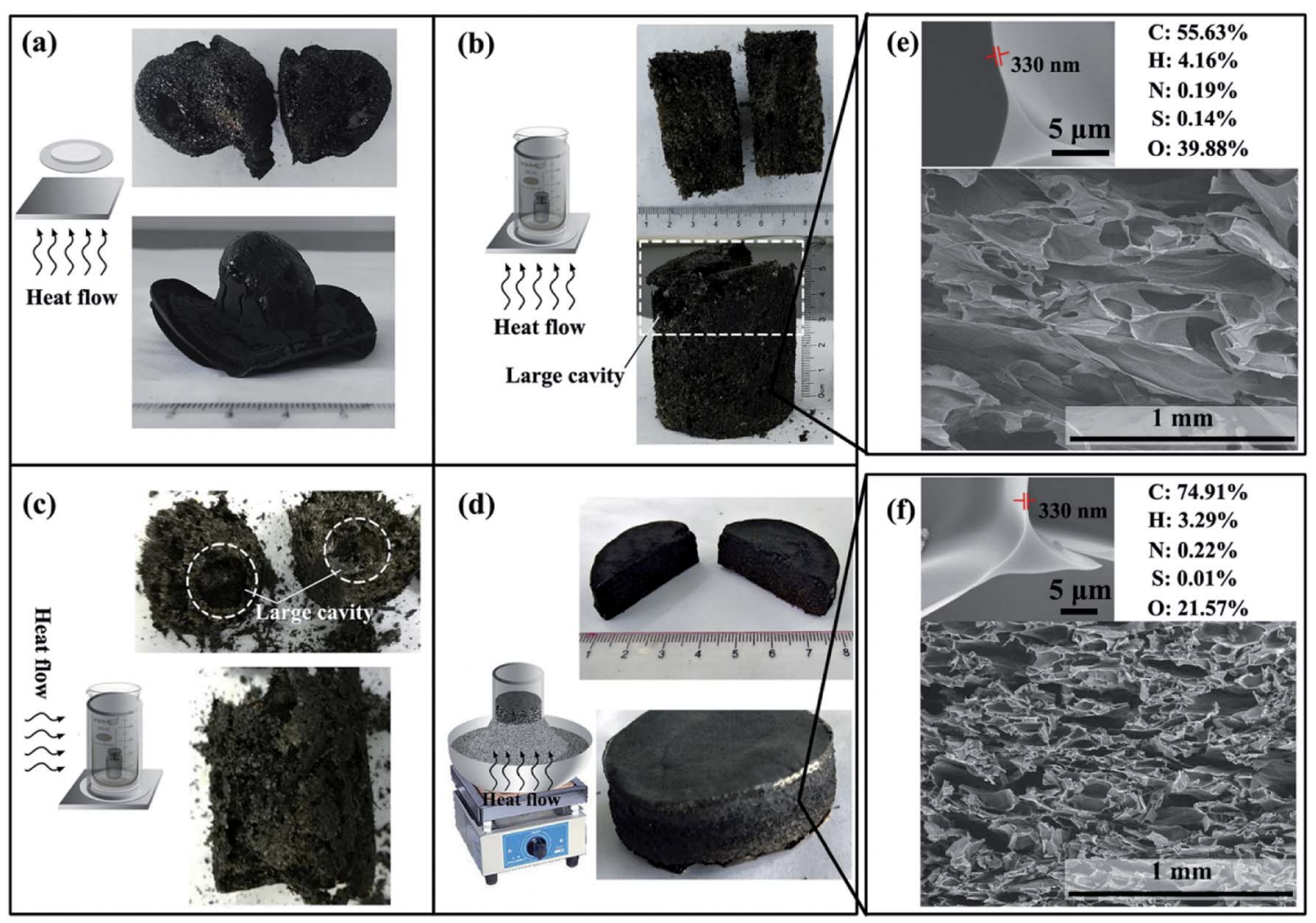

Fig. 6 The effect of pressure, exhaust and heat transfer on the preparation of CF. 
(a)

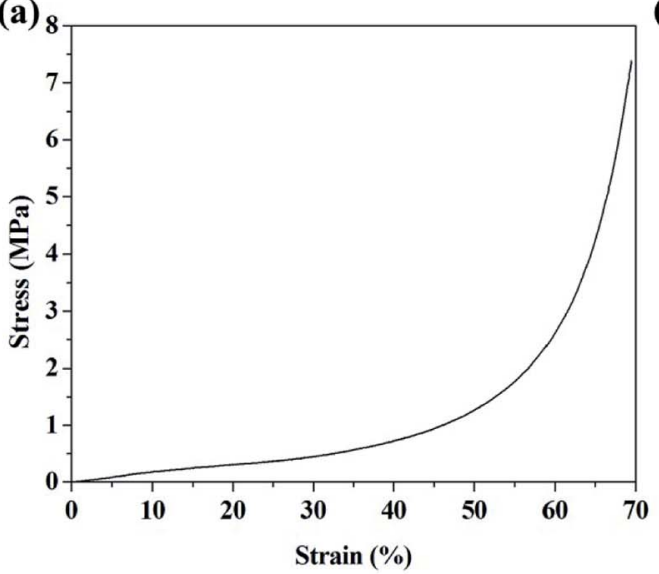

(b)

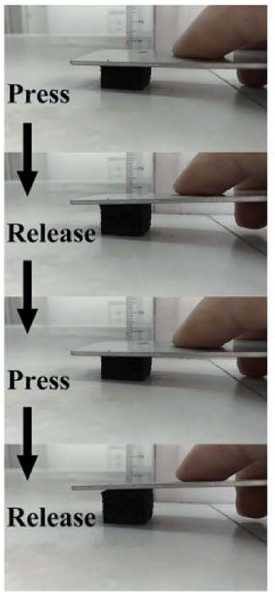

(c)

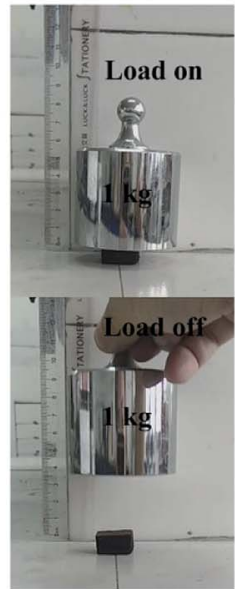

Fig. 7 (a) The compressive stress-strain curve of the CF in Fig. 6d; (b) and (c): the CF is compressible with good mechanical property.

CF prepared by electric furnace has more compact cells. This is caused by their different heating programs during preparation. The faster heating rate in Fig. $6 \mathrm{~d}$ enables the product to be carbonized and fixed in time without being over-foamed, so that no large cavities appear and the product is more compact. According to the enlarged SEM images in Fig. 5e and f, the CFs prepared in both ways have submicron ultrathin cell walls. However, the product obtained at higher temperature has a higher carbon content in its structure.

\section{Modification and oil/water separation}

Considering the brittleness of conventional carbon foam is a severe threat to its application in oil/water separation, we examined the compressive property of the CF shown in Fig. 6d. Its compressive stress-strain curve in Fig. 7a shows the characteristics of a typical elastic material, ${ }^{35}$ indicating that the $\mathrm{CF}$ is compressible. Fig. $7 \mathrm{~b}$ and $\mathrm{c}$ and our ESI Videos $\dagger$ also proved that the obtained CF has good mechanical property.

The pristine starch-derived CF was hydrophobic with water contact angle of about $142^{\circ}$ (inset of Fig. 8a). This value was greatly improved after modification with HMDS. It reached to $167^{\circ}$ (inset of Fig. 8c). The corresponding SEM images in Fig. 8a and $\mathrm{c}$ show that the morphology of the CF didn't change too much after modification. The difference can only be seen under high magnification. As shown in Fig. $8 \mathrm{~b}$ and $\mathrm{d}$, the surface of the pristine carbonaceous walls is smooth. But some wrinkles appeared on the walls after modification. According to the
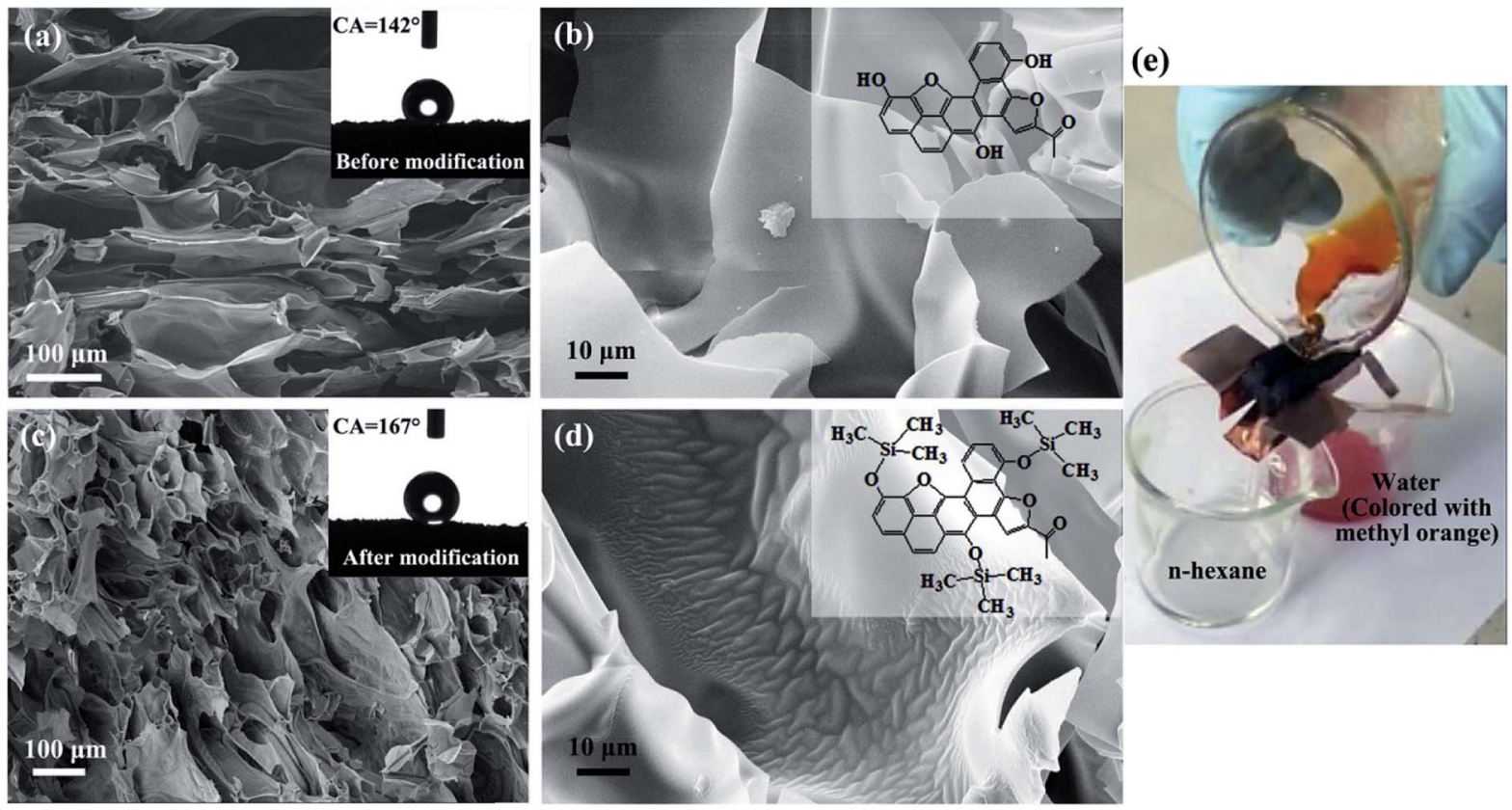

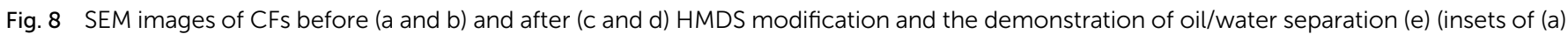

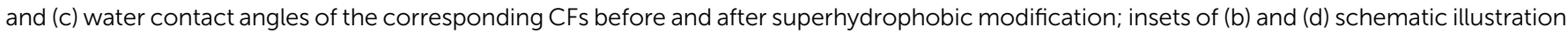
of the superhydrophobic modification of CF). 
coverage of the wrinkles, the surface of the carbonaceous walls has been uniformly modified. The modification of methyl groups to the surface of the CF is schematically shown in the insets of Fig. $8 \mathrm{~b}$ and $\mathrm{d}$.

The modified CF was then applied in oil/water separation. Due to the stratified distribution of the cells of CF, it should be ensured that the flow direction of the liquid (the oil phase in our case) is consistent with the layered texture when the CF is cut, so that the liquid (i.e., the oil phase) can easily pass though it. Fig. 8e and our ESI Video $\dagger$ demonstrate that the modified CF performs well in $n$-hexane/water separation. It shows the potential for application in the treatment of oil spill accidents and industrial discharge of oily wastewater.

\section{Conclusions}

The transition mechanism from starch to a novel compressible CF was studied in this work. The enlightenment on the preparation of CFs from different biomass raw materials was also summarized based on the research. The acids dispersion in the molten starch, decomposition gases release from the acidsstarch reaction, and carbonization under the effect of acid dehydration are analogy to the implementation of gas, expansion of gas, and stabilization of the polymer in the preparation of polymer foam, respectively. Acids can reduce the melting temperature of starch and broaden the carbonization temperature interval. Different from the brittleness of many conventional CFs, the as-prepared CF in this work is compressible. After superhydrophobic modification, the water contact angle of the material reached $167^{\circ}$, indicating its potential for application in oil/water separation.

\section{Conflicts of interest}

There are no conflicts to declare.

\section{Acknowledgements}

The authors would like to acknowledge the financial support by National Natural Science Foundation of China (No. 51602267) and Longshan academic talent research supporting program of SWUST (17LZX601; 18LZX547). Besides, the author Hong Lei wants to express his love to his wife (Zhizhi Wang) and thank her for her encouragement and support in this research.

\section{References}

1 H. Liu, L. Zhang, N. Wang and D. Su, Angew. Chem., Int. Ed., 2014, 53, 12634.

2 H. Liu, J. Wang, Z. Feng, Y. Lin, L. Zhang and D. Su, Small, 2015, 11, 5059.

3 J. Diao, Y. Zhang, J. Zhang, J. Wang, H. Liu and D. Su, Chem. Commun., 2017, 53, 11322.

4 F. Huang, H. Liu and D. Su, Sci. China Mater., 2017, 60, 1149.

5 M. Letellier, A. Szczurek, M. C. Basso, A. Pizzi, V. Fierro, O. Ferry and A. Celzard, Carbon, 2017, 112, 208.

6 P. Zhou and Q. Chen, J. Anal. Appl. Pyrolysis, 2016, 122, 370.
7 M. D. Sarzynski, MS thesis, Texas A\&M University, 2003.

8 J. Seo, H. Park, K. Shin, S. H. Baeck, Y. Rhym and S. E. Shim, Carbon, 2014, 76, 357.

9 J. L. Song, Y. L. Zhao, X. J. He, D. S. Zhang, Z. C. Tao, Q. G. Guo, X. Q. Gao, J. L. Shi, L. Liu, H. H. Xia and X. T. Zhou, Mater. Sci. Technol., 2013, 29, 1389.

10 Y. Wang, Z. He, L. Zhan and X. Liu, Mater. Lett., 2016, 169, 95.

11 N. Díez, C. Botas, E. Goikolea and D. Carriazo, J. Mater. Sci., 2017, 52, 11191.

12 R. Narasimman, S. Vijayan and K. Prabhakaran, Mater. Lett., 2015, 144, 46.

13 R. Narasimman, S. Vijayan, K. S. Dijith, K. P. Surendran and K. Prabhakaran, Mater. Chem. Phys., 2016, 181, 538.

14 P. Wilson, S. Vijayan and K. Prabhakaran, Carbon, 2017, 118, 545.

15 P. Wilson, S. Vijayan and K. Prabhakaran, Mater. Des., 2018, 139, 25.

16 Y. Yao, F. Chen, X. Chen, Q. Shen and L. Zhang, Diamond Relat. Mater., 2016, 64, 153.

17 G. Tondi, V. Fierro, A. Pizzi and A. Celzard, Carbon, 2009, 47, 1480.

18 P. Navard, Cellulose, 2005, 12, 337.

19 M. Bakierska, M. Molenda, D. Majda and R. Dziembaj, Procedia Eng., 2014, 98, 14.

20 J. Duan, C. Yu, L. Fan, M. Chen and J. Qiu, New Carbon Mater., 2013, 28, 178.

21 A. R. Rutledge, R. A. Venditti, J. J. Pawlak, S. Patel and J. L. Cibils, BioResources, 2008, 3, 1063.

22 M. Priyanka and M. P. Saravanakumar, J. Cleaner Prod., 2018, 197, 511.

23 S. Li, Y. Tian, Y. Zhong, X. Yan, Y. Song, Q. Guo, J. Shi and L. Liu, Carbon, 2011, 49, 618.

24 M. Inagaki, J. Qiu and Q. Guo, Carbon, 2015, 87, 128.

25 R. G. Utrilla-Coello, C. Hernández-Jaimes, H. Carrillo-Navas, F. González, E. Rodríguez, L. A. Bello-Pérez, E. J. VernonCarter and J. Alvarez-Ramirez, Carbohydr. Polym., 2014, 103, 596.

26 A. Turki, A. E. Oudiani, S. Msahli and F. Sakli, Carbohydr. Polym., 2018, 186, 226.

27 H. Lei, Y. Wang and J. Huo, Microporous Mesoporous Mater., 2015, 210, 39.

28 H. Lei and X. Cao, J. Mater. Sci., 2015, 50, 5318.

29 S. T. Lee, in Polymeric foams: mechanisms and materials, ed. S.

T. Lee and N. S. Ramesh, CRC, Boca Raton, 2004, vol. 1, p. 6. 30 R. Wang, W. Li and S. Liu, J. Mater. Sci., 2012, 47, 1977.

31 R. Mohammadinejad, S. Karimi, S. Iravani and R. S. Varma, Green Chem., 2016, 18, 20.

32 C. Chen, J. Song, S. Zhu, Y. Li, Y. Kuang, J. Wan, D. Kirsch, L. Xu, Y. Wang, T. Gao, Y. Wang, H. Huang, W. Gan, A. Gong, T. Li, J. Xie and L. Hu, Chem, 2018, 4, 544.

33 H. Sun, Z. Xu and C. Gao, Adv. Mater., 2013, 25, 2554.

34 P. H. Hermans and A. Weidinger, J. Appl. Phys., 1948, 19, 491. 35 B. M. Patterson, N. L. Cordes, K. Henderson, J. J. Williams, T. Stannard, S. S. Singh, A. R. Ovejero, X. Xiao, M. Robinson and N. Chawla, J. Mater. Sci., 2016, 51, 171. 